\title{
EPD: Restart your computer to finish important updates
}

\section{Yvonne Gilli}

Dr. med., Mitglied des FMH-Zentralvorstandes, Departement Digitalisierung / eHealth

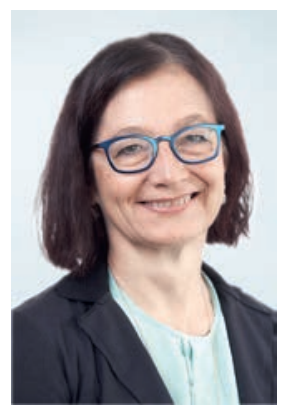

In der Schweiz ist die sekundäre Nutzung von Daten des elektronischen Patientendossiers (EPD) nicht vorgesehen und bedarf einer gesetzlichen Regelung auf kantonaler Ebene. Dabei bestünde gerade im Zusammenhang mit dem Swiss Personalized Health Network (SPHN) ein grosses Potenzial für Synergien [1]. Während hierzulande derzeit die letzten Hürden für die Einführung des EPD aus dem Weg geräumt werden, sind andere Länder einen Schritt weiter [2] und nutzen diese Daten bereits zu Forschungszwecken. Kanada hat dieses Jahr angekündigt, einen systematischen und transparenten Ansatz für die Nutzung von Real World Data (RWD) während des gesamten Lebenszyklus eines Arzneimittels zu entwickeln [3]. Der konstante Einbezug von RWD wird es ermöglichen, das Verständnis über die öffentliche Gesundheit zu steigern, die Gesundheitsversorgung zu verbessern sowie den Zugang zu Medikamenten für bestimmte Patientengruppen zu erleichtern.

Auch Australien ermöglicht in «My Health Record» bereits seit 2012 die Weitergabe von Daten für Forschungszwecke [4]. Angesichts der initialen Investitionskosten von etwa 500 Millionen Australischen Dollar [5] und jährlichen Folgekosten von mehreren hundert Millionen Australischen Dollar erscheint eine Partizipationsrate von nur $20 \%$ der Bevölkerung in den ersten fünf Jahren eher tief. Seit Anfang 2019 müssen Patientinnen und Patienten deshalb einer Teilnahme aktiv widersprechen (Opt-out). Durch diese Massnahme hat sich die Partizipationsrate auf 90\% der australischen Bevölkerung erhöht.

Für die Schweizer Bevölkerung ist die Teilnahme am EPD nach wie vor fakultativ (Opt-in), wobei Erfahrungen aus dem Kanton Genf zeigen, dass hierzulande mit einer tiefen Partizipationsrate von unter 10\% gerechnet werden muss [6]. Für die erfolgreiche Implementierung des EPD mit einer hohen Anzahl an Nutzerinnen und Nutzern werden also auch in der Schweiz unter Umständen mehrere Anläufe nötig sein. Unter anderem, weil das EPD nicht als direktes Kommunikationsmedium konzipiert worden ist und auch nicht zu einer Verbesserung der Effizienz im Gesundheitswesen führen wird: Für die Einsichtnahme in das EPD bedarf es einer aktiven $\mathrm{Zu}$ stimmung der Patientinnen und Patienten, die im Ein- zelfall und ad personam entscheiden müssen, welche Daten sie mit ihren Gesundheitsfachpersonen teilen möchten. In diesem Umfeld entstehen derzeit Zusatzdienste, die den Kommunikationsbedürfnissen der Gesundheitsfachpersonen entsprechen. Darüber hinaus bieten diese Zusatzdienste eine echte Prozessunterstützung und tragen damit zu einer effizienteren Gesundheitsversorgung bei. Selbstredend müssen die im EPD erfassten Daten und Zusatzdienste tief in die Primärsysteme integriert werden, um den administrativen Aufwand spürbar zu reduzieren.

\section{Die Datennutzung des EPD bietet grosses}

Synergiepotenzial - zum Beispiel in Zusammenhang mit dem Swiss Personalized Health Network.

Aktuell sieht es danach aus, dass das EPD kurz vor seinem Start bereits ein erstes Systemupdate erfährt. Im Parlament spricht sich derweil eine deutliche Mehrheit dafür aus, ambulant tätige Gesundheitsfachpersonen zum EPD zu verpflichten. Um auf diese Entwicklung zugunsten der Ärzteschaft Einfluss nehmen zu können, wird sich die FMH an der Firma AD Swiss Net AG beteiligen. Die AD Swiss Net AG, zu gleichen Teilen von der Ärztekasse und der Health Info Net AG (HIN) gegründet, wird als Gemeinschaft eine Plattform für den digitalen Austausch medizinischer Daten im Sinne des EPD anbieten. Mit ihrer Beteiligung stellt die FMH sicher, dass die angebotenen Dienstleistungen zu den medizinischfachlichen Anforderungen der Ärzteschaft und zu den gesundheitspolitischen Interessen der FMH passen.

Literatur

1 Meystre SM, Lovis C, Bürkle T, Tognola G, Budrionis A, Lehmann CU. Clinical Data Reuse or Secondary Use: Current Status and Potential Future Progress. Yearbook of medical informatics. 2017

2 Prokosch H-U, Acker T, Bernarding J, Binder H, Boeker M, Boerries $M$, et al. MIRACUM: Medical Informatics in Research and Care in University Medicine. Methods Inf Med. 2018.

3 https://www.canada.ca/en/health-canada/services/drugs-healthproducts/drug-products/announcements/optimizing-real-worldevidence-regulatory-decisions.html

4 Australian Department of Health. Framework to guide the secondary use of My Health Record system data. 2018;(May).

5 https://www.health.gov.au/internet/budget/publishing.nsf/ Content/budget2010-hmedia09.htm

6 MonDossierMedical [Internet]. [cited 2019 Jul 2]. Available from: https://www.mondossiermedical.ch/historique 